\title{
Effectiveness of adenoidectomy in children with recurrent upper respiratory tract infections: open randomised controlled trial
}

\author{
(C) $(1) \Theta$ OPEN ACCESS
}

\begin{abstract}
M T A van den Aardweg PhD student and registrar in otorhinolaryngology ${ }^{1}, \mathrm{C}$ W B Boonacker PhD student and clinical epidemiologist ${ }^{2}, \mathrm{M}$ M Rovers associate professor of clinical epidemiology ${ }^{12}, \mathrm{~A}$ W Hoes professor of clinical epidemiology and general practice ${ }^{2}$, A G M Schilder professor of paediatric otorhinolaryngology; ear, nose, and throat surgeon; and clinical epidemiologist ${ }^{12}$
\end{abstract}

${ }^{1}$ Department of Otorhinolaryngology, University Medical Centre Utrecht, Netherlands; ${ }^{2}$ Julius Centre for Health Sciences and Primary Care, University Medical Centre Utrecht

\begin{abstract}
Objective To assess the effectiveness of adenoidectomy in children with recurrent upper respiratory tract infections.

Design Open randomised controlled trial.

Setting 11 general hospitals and two academic centres.

Participants 111 children aged 1-6 with recurrent upper respiratory tract infections selected for adenoidectomy.

Intervention A strategy of immediate adenoidectomy with or without myringotomy or a strategy of initial watchful waiting.

Main outcome measure Primary outcome measure: number of upper respiratory tract infections per person year calculated from data obtained during the total follow-up (maximum 24 months). Secondary outcome measures: days with upper respiratory tract infection per person year, middle ear complaints with fever in episodes and days, days with fever, prevalence of upper respiratory tract infections, and health related quality of life.

Results During the median follow-up of 24 months, there were 7.91 episodes of upper respiratory tract infections per person year in the adenoidectomy group and 7.84 in the watchful waiting group (difference in incidence rate $0.07,95 \%$ confidence interval -0.70 to 0.85 ). No relevant differences were found for days of upper respiratory tract infections and middle ear complaints with fever in episodes and days, nor for health related quality of life. The prevalence of upper respiratory tract infections decreased over time in both groups. Children in the adenoidectomy group had significantly more days with fever than the children in the watchful waiting group. Two children had complications related to surgery.
\end{abstract}

Conclusion In children selected for adenoidectomy for recurrent upper respiratory tract infections, a strategy of immediate surgery confers no clinical benefits over a strategy of initial watchful waiting.

Trial registration Dutch Trial Register NTR968: ISRCTN03720485.

\section{Introduction}

An acute upper respiratory tract infection is the most common diagnosis in children in primary care: every year the diagnosis is made in one in every two children aged $0-4$ and in one in 10 of those aged 5-9. ${ }^{1}$ The true incidence of the condition in the community is much higher as usually parents do not consult their doctor when their child develops an upper respiratory tract infection. Upper respiratory tract infections not only affect children's health but also account for a large proportion of annual healthcare expenditure and high indirect costs for the family and society. ${ }^{2-4}$

An estimated $20 \%$ of children experience recurrent upper respiratory tract infections, and many of these children are referred to the ear, nose, and throat surgeon for surgery. ${ }^{5-8}$ Adenoidectomy is one of the most commonly performed surgical procedures in children in western countries. In 2009 in the Netherlands 15179 children (16.3 per 1000) aged 0-4 and 5573 children (5.5 per 1000) aged 5-9 underwent adenoidectomy. ${ }^{9}{ }^{10}$ In $60 \%$ of these children, recurrent upper respiratory tract infections were the indication for surgery. ${ }^{11}$ In 2006 in the United States 129540 children (1.76 per 1000) up to the age of 18 underwent adenoidectomy. In $12 \%$ of these children the operation was performed because of chronic infections. ${ }^{12}$ In 
both countries the figures have remained stable over the past decade. $^{1213}$

Remarkably, the adenoidectomy rate is more than three times higher in the Netherlands than in the US, and the proportion of children operated on for infections varies fivefold across these two countries, suggesting that there is no international consensus as to which children benefit from the operation.

Evidence for the effectiveness of adenoidectomy in children with recurrent upper respiratory tract infections is indeed scarce and (inter)nationally accepted guidelines are lacking. In our recent Cochrane review we showed that so far only two randomised controlled trials of adenoidectomy in children included upper respiratory tract infections as an outcome measure. ${ }^{14}$ One study was methodologically weak, ${ }^{15}$ and the other was performed in children with recurrent acute otitis media rather than upper respiratory tract infections. ${ }^{16}$ In this open multicentre randomised controlled trial we studied the effectiveness of adenoidectomy in children with recurrent upper respiratory tract infections.

\section{Methods}

\section{Patients}

We performed an open multicentre randomised controlled trial between April 2007 and October 2010. Ear, nose, and throat surgeons in 11 general hospitals and two academic centres were asked to complete a questionnaire on their patients aged 1-6 whom they selected for adenoidectomy with or without myringotomy. They were asked to list the indication for the operation and any previous ear, nose, and throat surgery. Parents who had expressed interest in the trial were contacted by a member of our study team. Children were eligible to participate in the trial if they were selected for adenoidectomy for recurrent upper respiratory tract infections. The parents were given detailed information about the trial, exclusion criteria were checked, and a standard demographic and disease specific questionnaire was completed. We excluded children who had previously undergone adenoidectomy or adenotonsillectomy and those with tympanostomy tubes (grommets) or who had an indication for insertion of tympanostomy tubes. We also excluded children with Down's syndrome and craniofacial malformation.

\section{Randomisation}

Children whose parents gave informed consent were randomly assigned to one of two strategies: adenoidectomy with or without myringotomy within six weeks or initial watchful waiting. For this purpose we used a computerised minimisation strategy - that is, a method of ensuring balance between prognostic factors in small samples ${ }^{17}$; factors that were taken into account were age $(<2$ and $\geq 2)$ and hospital. Treatment allocation was concealed until formal informed consent was obtained and the child was included in the trial.

\section{Baseline measurements}

When children entered the study, the study doctor filled in a demographic and disease specific questionnaire including information on the number of upper respiratory tract infections in the year before trial entry, previous ear, nose, and throat operations, and risk factors for upper respiratory tract infections. Parents filled in two generic and three disease specific questionnaires on health related quality of life: the child health questionnaire, ${ }^{18} 19$ the RAND general health rating index for children, ${ }^{20}{ }^{21}$ the sinonasal symptoms questionnaire, ${ }^{22}$ the OSA-18 quality of life questionnaire, ${ }^{23}$ and the otitis media- 6 questionnaire. ${ }^{24}$ All children underwent an ear, nose, and throat examination including fibreoptic endoscopy of the nasopharynx. Adenoid size was graded as obstructing the choanae for $0-25 \%$, $26-50 \%, 51-75 \%$, or $76-100 \%$. A blood sample was taken for the Phadiatop test, an allergen specific IgE test to a panel of common food and aeroallergens in children, with the result classified as positive or negative. Finally, data on nasopharyngeal flora, exhaled nitric oxide, and costs were collected at baseline and during follow-up. These results will be reported separately.

\section{Follow-up}

During the two year follow-up parents kept a diary, including specific symptoms of upper respiratory tract infections: nasal stuffiness, mouth breathing, nasal discharge, sore throat, cough, and fever. They also noted middle ear complaints and absence from day care or school because of upper respiratory tract infections. They measured their child's temperature every day with a validated tympanic membrane thermometer. To avoid information bias, we had an electronic device built in, which stored date and first temperature measurement of each day. ${ }^{25}$ The study doctor collected the diary and thermometer data during the follow-up visits at 3,6,12, 18, and 24 months and examined the child's ears, nose, and throat. At those visits parents also filled in questionnaires on health related quality of life.

Parents, general practitioners, and ear, nose, and throat surgeons of the participating children were encouraged to manage upper respiratory tract infections during follow-up according to their regular practice.

\section{Primary and secondary outcomes}

The primary outcome measure was the number of upper respiratory tract infections per person year calculated from data obtained during follow-up (maximum 24 months). The definition of upper respiratory tract infection was two or more of the following: fever (a temperature of $38^{\circ} \mathrm{C}$ or higher as measured by a tympanic thermometer), diary scored symptoms of nasal stuffiness or mouth breathing, nasal discharge, sore throat, or cough. An episode ended when the child was free from symptoms for at least a day. A new episode was recorded after at least seven days without symptoms or fever.

Secondary outcome measures were days with upper respiratory tract infection per person year, incidences of mild and severe upper respiratory tract infection, and middle ear complaints with fever in episodes and days, days with fever, days of absence from day care or school because of upper respiratory tract infection, prevalence of upper respiratory tract infections, and health related quality of life. Mild upper respiratory tract infection was defined as an infection without fever and resolving within 10 days. Severe upper respiratory tract infection was defined as an infection persisting for more than 10 days or an infection accompanied by fever. Middle ear complaints were defined as acute otorrhoea, earache, or pulling the ear accompanied by fever. To measure the burden of upper respiratory tract infections during follow-up we calculated the prevalence of upper respiratory tract infections per week. Generic health related quality of life was assessed with the child health questionnaire ${ }^{18} 19$ and the RAND general health rating index for children ${ }^{20}{ }^{21}$ and disease specific health related quality of life with the sinonasal symptoms questionnaire, ${ }^{22}$ the OSA-18 quality of life questionnaire, ${ }^{23}$ and the otitis media-6 questionnaire. $^{24}$ 


\section{Statistical analysis}

Our sample size calculation was based on a clinically relevant reduction of upper respiratory tract infections of 33\%. Assuming a mean baseline incidence of six (SD three) upper respiratory tract infections each year, and taking $\alpha=0.05$ and a power of 0.90 , we calculated that we would need 49 children in each group. To allow for $10 \%$ loss to follow-up we aimed to include 110 children.

The effects of adenoidectomy on upper respiratory tract infection episodes and days were calculated as differences in incidence rates and incidence rate ratios per person year with $95 \%$ confidence intervals. Scores on health related quality of life instruments were linearly transformed into $0-100$ scales (100 being the best possible score) and presented per subscale. We used Student's $t$ tests or Mann-Whitney U tests to evaluate differences between the two groups. Poisson regression analyses with a robust covariance matrix estimator were used to adjust for potential confounding (observed baseline differences in prognostic factors, such as sex, breast feeding for more than three months, family history of upper respiratory tract infections, and passive smoking). The $95 \%$ confidence intervals of the adjusted rate differences and ratios were addressed in $\mathrm{R}$ by means of bootstrapping, for which we replicated the trial 10000 times using random replacement samples.

Potential modification of the effect of adenoidectomy was evaluated with Poisson analyses including interaction terms for age $(<2$ and $\geq 2)$, adenoid size $(\leq 75 \% v>75 \%$ obstruction of the choanae), and Phadiatop (positive $v$ negative). Subgroups were further analysed only in case of significant interaction effects.

In addition to the intention to treat analysis, we also performed two sensitivity analyses: a per protocol analysis in which we excluded the children in the watchful waiting group who went on to have surgery, and an as treated analysis in which we added the children in the watchful waiting group who underwent surgery to the adenoidectomy group.

To study the external validity, we compared demographic and disease specific characteristics of the included children with those who were eligible to participate but whose parents did not give informed consent. We used Pearson's $\chi^{2}$ tests to compare these characteristics.

All analyses were performed according to the intention to treat principle with SPSS version 17 (SPSS, Chicago, IL), Rothman's Episheet (11 June 2008), and R version 2.13.0 (13 April 2011).

\section{Results}

\section{Patients}

Between April 2007 and April 2009, 373 children aged 1-6 selected for adenoidectomy for recurrent upper respiratory tract infections were referred to our trial centre. Of these, $262(70 \%)$ were ineligible or excluded for various reasons (fig $1 \Downarrow$ ), and 111 were randomly assigned to one of two strategies: 54 children to adenoidectomy with or without myringotomy within six weeks and 57 children to initial watchful waiting.

Table 1 shows the baseline characteristics. $\Downarrow$ The mean age was 36 and 38 months and the median number of upper respiratory tract infection episodes in the year before trial entry was 10 in the adenoidectomy group and nine in the watchful waiting group. Median follow-up was 24 months in both groups.

During the trial period, 11 (10\%) children were lost to follow-up for non-medical reasons: four (7\%) from the adenoidectomy group and seven (12\%) from the watchful waiting group. All children allocated to adenoidectomy underwent surgery within six weeks: 48 (89\%) had adenoidectomy alone and six (11\%) had adenoidectomy and myringotomy. During follow-up seven (13\%) children allocated to adenoidectomy underwent tonsillectomy and revision adenoidectomy and three $(6 \%)$ had tympanostomy tubes inserted. During follow-up 23 (40\%) children allocated to watchful waiting underwent further surgery. Seventeen underwent adenoidectomy (in 11 (19\%) it was adenoidectomy only; in four (7\%) it was combined with myringotomy and in two (4\%) with tympanostomy tubes; one (2\%) child underwent adenoidectomy at 12 months and revision adenoidectomy with tympanostomy tubes at 24 months, one (2\%) underwent adenoidectomy at six months and tonsillectomy at 12 months). Six (11\%) underwent adenotonsillectomy (in one combined with myringotomy).

\section{Primary outcome}

During the total follow-up the incidences of upper respiratory tract infections episodes in the adenoidectomy and watchful waiting group were 7.91 and 7.84 per person year (difference in incidence rate $0.07,95 \%$ confidence interval -0.70 to 0.85 ; table $2 \Downarrow$ ). These incidences were 9.22 and 9.39 per person year (difference $-0.17,-1.34$ to 1.00 ), respectively, during the first year of follow-up and 6.55 and 6.17 per person year (difference $0.37,-0.62$ to 1.37 ), respectively, during the second year of follow-up (table $3 \Downarrow$ ). Similar results were found after adjustment for observed baseline differences - that is, the adjusted rate differences for the total follow-up, year one, and year two were -0.03 ( -1.72 to 1.67$),-0.14$ ( -1.76 to 1.68$)$, and $0.13(-2.05$ to 2.32 ) (table $4 \Downarrow$ ).

\section{Secondary outcomes}

During the total follow-up there were 66.10 and 67.36 days with upper respiratory tract infection per person year (difference in incidence rate $-1.27,-3.52$ to 0.99 ; table $2 \Downarrow)$ in the adenoidectomy and watchful waiting group, respectively.

Figure $2 \Downarrow$ shows that the proportion of children with an upper respiratory tract infection (expressed as the prevalence per week) decreased over time in both groups. No differences were found between the two groups for episodes of mild and severe upper respiratory tract infection and days per person year during the total follow-up (table $2 \Downarrow$ ). Children in the adenoidectomy group had significantly more days with fever than the children in the watchful waiting group: $20.00 v 16.49$ days per person year during the total follow-up (difference 3.51, 2.33 to 4.69).

During the total follow-up there were 0.51 episodes of middle ear complaints with fever per person year in the adenoidectomy group and 0.45 in the watchful waiting group (difference 0.05 , -0.14 to 0.24 (table $2 \Downarrow$ ). Children in the adenoidectomy group had 0.86 days per person year of middle ear complaints with fever and children in the watchful waiting group had $0.85(0.01$, -0.24 to 0.27 ). Days of absence from day care or school because of an upper respiratory tract infection were 1.66 and $2.00(-0.33$, -0.71 to 0.04 ) in the adenoidectomy and watchful waiting groups, respectively. Table $3 \Downarrow$ shows the results for follow-up year one and two separately. After adjustment for observed baseline differences, we found no significant differences (table $4 \Downarrow)$.

Health related quality of life as measured by generic (child health questionnaire, ${ }^{18} 19$ and RAND ${ }^{20}{ }^{21}$ ) and disease specific (sinonasal symptoms questionnaire, ${ }^{22}$ OSA-18 quality of life questionnaire, ${ }^{23}$ and otitis media- 6 questionnaire. ${ }^{24}$ ) questionnaires, did not differ significantly between both groups 
over time. Exact numbers are available in appendix 1 on bmj.com)

As we found no significant interaction terms, we did not further analyse any subgroups of patients.

\section{Crossovers}

We found no significant differences in baseline variables nor in the number of upper respiratory tract infections during the first year of follow-up between those children in the control group who did and did not cross over (data not shown).

The per protocol and as treated analyses (table $5 \Downarrow$ ) yielded the same results as the intention to treat analysis regarding our primary outcome - that is, upper respiratory tract infection episodes during the total follow-up. For example, the difference in incidence rate for episodes of upper respiratory tract infection was $-0.13(-1.02$ to 0.77$)$ for the per protocol analysis and -0.23 ( -1.08 to 0.62$)$ for the as treated analysis. The adjusted incidence rate differences also showed no significant differences for the primary outcome (table 6 ). $\Downarrow$

\section{Generalisability}

To assess the external validity of our results we compared demographic and disease specific characteristics of the children participating in the trial with those of the $165(60 \%)$ children who were eligible for the trial but did not participate for various reasons. In the trial participants and eligible but

non-participating children, respectively, the mean age at referral was 36 and 34 months, $59 \%$ and $56 \%$ were boys, $57 \%$ and $45 \%$ had symptoms of snoring or obstructive apnoea, $78 \%$ and $84 \%$ had nasal discharge on examination, and $67 \%$ and $69 \%$ had nasal obstruction on examination. Importantly, none of these variables differed significantly.

\section{Adverse events}

Two (4\%) children in the adenoidectomy group experienced an adverse event: one child was admitted to hospital for an asthma exacerbation during follow-up and in one child a primary tooth was broken when the mouth gag was inserted. One (2\%) child in the watchful waiting group who underwent adenotonsillectomy during follow-up was admitted to hospital for a postoperative haemorrhage.

\section{Discussion}

In children selected for adenoidectomy for recurrent upper respiratory tract infections, a strategy of immediate surgery did not reduce the number of upper respiratory tract infections compared with a strategy of initial watchful waiting. The prevalence of upper respiratory tract infections decreased similarly over time in both groups, suggesting that the contribution of surgery to the favourable natural course of upper respiratory tract infections is trivial.

We found no relevant differences between the two strategies for days of upper respiratory tract infections, days and episodes of mild and severe upper respiratory tract infections and middle ear complaints with fever, days of absence from day care or school, and health related quality of life. There was a significant difference for days with fever.

Forty per cent of children in the initial watchful waiting group underwent surgery during the course of the trial. These children, however, were not more severely affected by upper respiratory tract infections than the $60 \%$ who did not undergo surgery nor did they do better after surgery.

\section{Comparison with literature}

So far, most trials of adenoidectomy have been performed in children with recurrent acute otitis media or persistent otitis media with effusion and otitis media was studied as the primary outcome. These studies showed a benefit of adenoidectomy regarding the resolution of middle ear effusion and also a small benefit regarding hearing but did not observe a beneficial effect on recurrence of acute otitis media. ${ }^{26}$

The one study $(n=76)$ that did include children selected for adenoidectomy because of frequent upper respiratory tract infections showed that at 12 months' follow-up $75 \%$ of children in the adenoidectomy group and $73 \%$ of children in the control group improved during follow-up regarding common colds (risk difference $2 \%, 95 \%$ confidence interval $-18 \%$ to $22 \%$ ). At 24 months' follow-up these figures were $77 \%$ and $88 \%$, respectively $(-11 \%,-28 \%$ to $7 \%) .{ }^{15}$ Another study $(\mathrm{n}=180)$ of adenoidectomy versus chemoprophylaxis and placebo in children with recurrent acute otitis media included days with rhinitis as a secondary outcome. Children in the adenoidectomy group had four fewer days with rhinitis during six months of follow-up than those in the control groups (95\% confidence interval -13 to 7 days). ${ }^{16}$

All trials of adenoidectomy performed so far have had methodological limitations. ${ }^{1426}$ Firstly, only three trials provided a power analysis and included adequate numbers. As the other trials included relatively few patients, their power might have been too low, leading to a type II error. Secondly, most studies had significant loss to follow-up. This can be associated with either good or poor outcome. Thirdly, three studies were analysed per protocol rather than by intention to treat. Per protocol analyses underestimate the treatment effect as in surgical trials only children in the watchful waiting group with persisting complaints can change treatment group, whereas children of the surgical group, who might experience similar complaints, cannot change treatment group. Fourthly, information bias might have been considerable because trials on adenoidectomy, as most surgical trials, cannot be performed in a true double blind fashion. Such bias will overestimate the effect of the intervention. None of the trials tried to minimise information bias by choosing an objective outcome measure, such as fever. Finally, the generalisability of the trials can be questioned as only a small proportion of children undergoing adenoidectomy were included in the trials.

\section{Possible limitations}

Our trial has several limitations. Firstly, we emphasise that we compared two strategies (immediate adenoidectomy or initial watchful waiting). As in other surgical trials, such as our previous study on adenotonsillectomy, ${ }^{27}$ the fact that some patients in the surgery group undergo additional surgical interventions and some patients in the watchful waiting group eventually undergo adenoidectomy mimics daily practice. ${ }^{28} 29$ This is part of the two strategies we compared. We studied whether the children in the control group who went on to undergo adenoidectomy were more severely affected than those who did not. There were no significant differences in baseline variables nor in the number of upper respiratory tract infections during the first year of follow-up between those children in the control group who did and did not cross over (data not shown). Furthermore, the per protocol and as treated analyses yielded the same results as the intention to treat analysis regarding our primary outcome - that is, the number of upper respiratory tract infections during the total follow-up. 
Secondly, we chose $33 \%$ as indicating a clinically relevant difference (in absolute terms a decrease from six to four upper respiratory tract infections a year) as the incidence of upper respiratory tract infections in young children is high and is known to decrease over time spontaneously. Nevertheless, we looked into the probability that, given our results, a difference of 20-25\% - that is, a difference of 1.5 upper respiratory tract infections a year-could have occurred. Looking at the confidence interval of the total follow-up, the value -1.5 is not within the $99 \%$ confidence interval, which means that we can also confidently rule out a difference of 1.5 episodes. Therefore, it seems unlikely that the results and conclusions would change if we had chosen another clinically relevant difference in our power calculation.

Thirdly, we question whether our results are generalisable to all children with recurrent upper respiratory tract infections. As we found no statistical differences between the trial participants and those eligible but non-participating, and none of the studied characteristics modified the effect of adenoidectomy, we think that these results are generalisable to all children selected for adenoidectomy for recurrent upper respiratory tract infections.

\section{Strengths of the study}

As our randomised controlled trial of adenoidectomy focused on children with recurrent upper respiratory tract infections, it provides important evidence for the many children selected for adenoidectomy for this indication. To our knowledge this is the first randomised controlled trial focusing specifically on these children. We included an objective method to study the effect of adenoidectomy - that is, fever measured daily by a validated thermometer that automatically stored data. Fever is an important physical sign in childhood infections, and most episodes of fever in young children aged under 8 are related to upper respiratory tract infections. ${ }^{30} 31$

For the randomisation process we applied a minimisation strategy that accounted for age and hospital. As such, we ensured that the children within each centre were equally distributed over the two groups. Therefore potential bias from possible differences in "traditions" of treating these children is precluded.

\section{Conclusion}

In children selected for adenoidectomy for recurrent upper respiratory tract infections, a strategy of immediate surgery confers no clinical benefits over a strategy of initial watchful waiting.

We thank the participants and their parents; Nelly van Eden for secretarial support, Nicole Boekema for data management, and Rolf Groenwold for assistance with the bootstrap analysis. We also thank our colleagues at the participating hospitals: Flevoziekenhuis Almere, Meander Medisch Centrum Amersfoort, Gelre Ziekenhuis Apeldoorn and Zutphen, Wilhelmina Ziekenhuis Assen, Albert Schweitzer Ziekenhuis Dordrecht, Universitair Medisch Centrum Groningen, Diakonessenhuis Meppel, St. Antonius Ziekenhuis Nieuwegein, Vlietland Ziekenhuis Schiedam, MESOS Medisch Centrum Utrecht and the Universitair Medisch Centrum Utrecht. Finally we thank the members of the executive steering committee: $\mathrm{E} H$ van den Akker, M J M Bonten, $K$ Fischer, E A M Sanders, and R van Weissenbruch.

Contributors: MTAvdA and CWBB collected, analysed, and interpreted the data, and wrote the paper. MMR designed, planned, and supervised the study, analysed and interpreted the data. AGMS and AWH designed, planned, and supervised the study and interpreted the data. The manuscript was prepared by MTAvdA and CWBB and commented on by all authors. AGMS is guarantor.
Funding: This study was funded by ZonMw-the Netherlands Organisation for Health Research and Development, healthcare efficiency research programme, subprogramme effects and costs 2007 No 80-007022-98-07901. The funder had no role in the study design and the collection, analysis, and interpretation of data and the writing of the article and the decision to submit it for publication. All authors were independent from the funder and had access to all the data. Competing interests: All authors have completed the ICMJE uniform disclosure form at www.icmje.org/coi_disclosure.pdf (available on request from the corresponding author) and declare: no support from any organisation for the submitted work; no financial relationships with any organisations that might have an interest in the submitted work in the previous three years; no other relationships or activities that could appear to have influenced the submitted work. AGMS and MMR have participated in workshops and educational activities on otitis media organised by GlaxoSmithKline and have received a grant from GlaxoSmithKline for a study on the microbiology of otitis media in 2009. Ethical approval: This study was approved by the medical ethics committees of the University Medical Centre Utrecht and all participating hospitals and was monitored according to Good Clinical Practice. Informed consent was obtained from all participants.

Data sharing: Statistical code and dataset are available from the corresponding author. Consent for data sharing was not obtained from participants, but the presented data are anonymised and risk of identification is low.

1 Nationaal Kompas Volksgezondheid. 2010. www.nationaalkompas.nl/gezondheid-enziekte/ziekten-en-aandoeningen/ademhalingswegen/infecties-van-de-bovenste-luchtwegen/ cijfers-infecties-van-de-bovenste-luchtwegen-incidentie-uit-de-vtv-2010/.

2 Carabin H, Gyorkos TW, Soto JC, Penrod J, Joseph L, Collet JP. Estimation of direct and indirect costs because of common infections in toddlers attending day care centers. Pediatrics 1999;103:556-64.

3 Dixon RE. Economic costs of respiratory tract infections in the United States. Am J Med 1985;78:45-51.

4 Harsten G, Prellner K, Heldrup J, Kalm O, Kornfalt R. Acute respiratory tract infections in children. A three-year follow-up from birth. Acta Paediatr Scand 1990;79:402-9.

5 Haapkyla J, Karevold G, Kvaerner KJ, Pitkaranta A. Trends in otitis media surgery: a decrease in adenoidectomy. Int J Pediatr Otorhinolaryngol 2008;72:1207-13.

6 Karevold G, Haapkyla J, Pitkaranta A, Kvaerner KJ. Otitis media surgery: large variability between Finland and Norway. Int J Pediatr Otorhinolaryngol 2007;71:1035-9.

7 Kvaerner KJ, Nafstad P, Jaakkola JJ. Otolaryngological surgery and upper respiratory tract infections in children: an epidemiological study. Ann Otol Rhinol Laryngol 2002;111:1034-9.

8 Schilder AG, Lok W, Rovers MM. International perspectives on management of acute otitis media: a qualitative review. Int J Pediatr Otorhinolaryngol 2004;68:29-36. CBS. Statline. 2011. http://statline.cbs.nl/statweb.

10 Prismant. Landelijke LMR-informatie-Verrichtingen. 2011. http://cognosserver.prismant. $\mathrm{nl} /$ cognos7/cgi-bin/ppdscgicgi?DC=Q\&E=/Prisma-Landelijke-LMR/Landelijke+LMRinformatie+-+Verrichtingen.

11 Van den Aardweg MT, Rovers MM, Kraal A, Schilder AG. Current indications for adenoidectomy in a sample of children in the Netherlands. B-ENT 2010;6:15-8.

12 Bhattacharyya N, Lin HW. Changes and consistencies in the epidemiology of pediatric adenotonsillar surgery, 1996-2006. Otolaryngol Head Neck Surg 2010;143:680-4.

13 Dutch Society for Otorhinolaryngology and Head and Neck Surgery. Guideline for adenoid and tonsil disorders [in Dutch]. 2007. www.cbo.nl/Downloads/283/rl_zatt_08.pdf.

14 Van den Aardweg MT, Schilder AG, Herkert E, Boonacker CW, Rovers MM. Adenoidectomy for recurrent or chronic nasal symptoms in children. Cochrane Database Syst Rev 2010;1:CD008282.

15 Rynnel-Dagoo B, Ahlbom A, Schiratzki H. Effects of adenoidectomy: a controlled two-year follow-up. Ann Otol Rhinol Laryngol 1978;87:272-8.

16 Koivunen P, Uhari M, Luotonen J, Kristo A, Raski R, Pokka T, et al. Adenoidectomy versus chemoprophylaxis and placebo for recurrent acute otitis media in children aged under 2 years: randomised controlled trial. BMJ 2004;328:487.

17 Altman DG, Bland JM. Treatment allocation by minimisation. BMJ 2005;330:843

18 Georgalas C, Tolley N, Kanagalingam J. Measuring quality of life in children with adenotonsillar disease with the child health questionnaire: a first UK study. Laryngoscope 2004;114:1849-55.

19 Wulffraat N, van der Net JJ, Ruperto N, Kamphuis S, Prakken BJ, Ten CR, et al. The Dutch version of the Childhood Health Assessment Questionnaire (CHAQ) and the Child Health Questionnaire (CHQ). Clin Exp Rheumatol 2001;19:S111-5.

20 Post MW, Kuyvenhoven MM, Verheij MJ, de Melker RA, Hoes AW. The Dutch "Rand general health rating index for children": a questionnaire measuring the general health status of children. Ned Tijdschr Geneeskd 1998;142:2680-3.

21 Post MW, Kuyvenhoven MM, Verheij MJ, de Melker RA, Hoes AW. The Dutch version of "functional status II(R)": a questionnaire measuring the functional health status of children. Ned Tijdschr Geneeskd 1998;142:2675-9.

22 Kay DJ, Rosenfeld RM. Quality of life for children with persistent sinonasal symptoms. Otolaryngol Head Neck Surg 2003;128:17-26.

23 Sohn H, Rosenfeld RM. Evaluation of sleep-disordered breathing in children. Otolaryngol Head Neck Surg 2003;128:344-52.

24 Rosenfeld RM, Goldsmith AJ, Tetlus L, Balzano A. Quality of life for children with otitis media. Arch Otolaryngol Head Neck Surg 1997;123:1049-54. 


\section{What is already known on this topic}

Adenoidectomy offers some benefits in children with otitis media

Recurrent upper respiratory tract infection is a common indication for adenoidectomy

Evidence of the benefits of adenoidectomy in children with recurrent upper respiratory tract infections is lacking

\section{What this study adds}

In children with recurrent upper respiratory tract infections adenoidectomy confers no clinical benefits over a strategy of watchful waiting

In children with recurrent upper respiratory tract infections the prevalence of such infections decreases over time irrespective of the treatment arm

Van Staaij BK, Rovers MM, Schilder AG, Hoes AW. Accuracy and feasibility of daily infrared tympanic membrane temperature measurements in the identification of fever in children. Int J Pediatr Otorhinolaryngol 2003;67:1091-7.

26 Van den Aardweg MT, Schilder AG, Herkert E, Boonacker CW, Rovers MM. Adenoidectomy for otitis media in children. Cochrane Database Syst Rev 2010;1:CD007810

27 Van Staaij BK, van den Akker EH, Rovers MM, Hordijk GJ, Hoes AW, Schilder AG. Effectiveness of adenotonsillectomy in children with mild symptoms of throat infection or adenotonsillar hypertrophy: open, randomised controlled trial. BMJ 2004;329:651.

28 Lock C, Wilson J, Steen N, Eccles M, Mason H, Carrie S, et al. North of England and Scotland study of tonsillectomy and adeno-tonsillectomy in children (NESSTAC): a pragmatic randomised controlled trial with a parallel non-randomised preference study. Health Technol Assess 2010;14:1-iv.

29 Paradise JL, Bluestone CD, Colborn DK, Bernard BS, Rockette HE, Kurs-Lasky M. Tonsillectomy and adenotonsillectomy for recurrent throat infection in moderately affected children. Pediatrics 2002;110:7-15.
30 Eskerud JR, Laerum E, Fagerthun H, Lunde PK, Naess A. Fever in general practice. I. Frequency and diagnoses. Fam Pract 1992;9:263-9.

31 Soman M. Characteristics and management of febrile young children seen in a university family practice. J Fam Pract 1985;21:117-22.

Accepted: 27 June 2011

\section{Cite this as: BMJ 2011;343:d5154}

This is an open-access article distributed under the terms of the Creative Commons Attribution Non-commercial License, which permits use, distribution, and reproduction in any medium, provided the original work is properly cited, the use is non commercial and is otherwise in compliance with the license. See: http://creativecommons.org/licenses/bync/2.0/ and http://creativecommons.org/licenses/by-nc/2.0/legalcode. 


\section{Tables}

Table 1| Baseline characteristics of 111 children with recurrent upper respiratory tract infections according to treatment allocation. Figures are numbers (percentage) unless stated otherwise

\begin{tabular}{|c|c|c|}
\hline & Adenoidectomy $(n=54)$ & Watchful waiting ( $n=57)$ \\
\hline \multicolumn{3}{|l|}{ Patients' characteristics } \\
\hline Mean (SD) age (months) & $36(19)$ & $38(18)$ \\
\hline Boys & $37(69)$ & $29(51)$ \\
\hline Breast fed $\geq 3$ months & $24(44)$ & $32(56)$ \\
\hline Positive result on Phadiatop test ${ }^{\star}$ & $12(24)$ & $16(30)$ \\
\hline Family history for recurrent URTIs & $31(57)$ & $41(72)$ \\
\hline Exposure to household nicotine smoke & $19(35)$ & $13(23)$ \\
\hline Children with household pets & $33(61)$ & $36(63)$ \\
\hline Children with siblings & $42(78)$ & $38(67)$ \\
\hline \multicolumn{3}{|l|}{ Education level of mother: } \\
\hline Low & $10(9)$ & $9(16)$ \\
\hline Average & $22(41)$ & $30(53)$ \\
\hline High & $22(41)$ & $18(32)$ \\
\hline Day care attendance aged $<4$ years & $33(81)$ & $36(88)$ \\
\hline \multicolumn{3}{|l|}{ Disease characteristics } \\
\hline Median No of episodes of URTI in year before trial entry (IQR) & 10 (3 to 17$)$ & 9 (2 to 17$)$ \\
\hline Median (IQR) OSA score $†$ & $-0.99(-2.41$ to 1.13$)$ & $-1.70(-2.42$ to 0.42$)$ \\
\hline Adenoid size $76-100 \%$ & $13(26)$ & $11(23)$ \\
\hline
\end{tabular}

IQR=interquartile range; URTI=upper respiratory tract infection; OSA=obstructive sleep apnoea.

${ }^{*}$ Allergen specific lgE test to panel of common food and aeroallergens.

†Brouilette OSA score: $1.42 \times$ difficulty breathing $+1.41 \times$ apnoea $+0.71 \times$ snoring-3.83. Range $-3.83-3.5$. Score $>3.5$ is highly predictive of OSA; score $-1-3.5$ indicates possible OSA; and score $<-1$ indicates no OSA. 
Table 2| Primary and secondary outcomes for total follow-up (maximum 24 months) in children with recurrent upper respiratory tract infections according to treatment allocation

\begin{tabular}{|c|c|c|c|c|}
\hline & $\begin{array}{l}\text { Adenoidectomy (101 } \\
\text { person years) }\end{array}$ & $\begin{array}{l}\text { Watchful waiting (101 } \\
\text { person years) }\end{array}$ & Difference in incidence rate $(95 \% \mathrm{Cl})$ & Incidence rate ratio $(95 \% \mathrm{Cl})$ \\
\hline \multicolumn{5}{|l|}{ Primary outcome: } \\
\hline URTI episodes & 7.91 & 7.84 & $0.07(-0.70$ to 0.85$)$ & $1.01(0.91$ to 1.11$)$ \\
\hline \multicolumn{5}{|l|}{ Secondary outcomes: } \\
\hline URTI days & 66.10 & 67.36 & $-1.27(-3.52$ to 0.99$)$ & $0.98(0.95$ to 1.01$)$ \\
\hline Severe URTI episodes & 3.98 & 3.53 & $0.45(-0.08$ to 0.99$)$ & $1.13(0.98$ to 1.30$)$ \\
\hline Severe URTI days & 48.11 & 46.56 & $1.55(-0.35$ to 3.44$)$ & $1.03(0.99$ to 1.08$)$ \\
\hline Mild URTI episodes & 3.93 & 4.31 & $-0.38(-0.94$ to 0.18$)$ & 0.91 (0.80 to 1.04$)$ \\
\hline Mild URTI days & 17.99 & 20.80 & $-2.81(-4.03$ to -1.60$)$ & 0.86 (0.81 to 0.92$)$ \\
\hline Fever days & 20.00 & 16.49 & 3.51 (2.33 to 4.69$)$ & $1.21(1.14$ to 1.29$)$ \\
\hline $\begin{array}{l}\text { Middle ear complaints with fever } \\
\text { episodes }\end{array}$ & 0.51 & 0.45 & 0.05 (-0.14 to 0.24$)$ & $1.11(0.75$ to 1.65$)$ \\
\hline $\begin{array}{l}\text { Middle ear complaints with fever } \\
\text { days }\end{array}$ & 0.86 & 0.85 & $0.01(-0.24$ to 0.27$)$ & 1.01 (0.75 to 1.36$)$ \\
\hline Absence from day care or school & 1.66 & 2.00 & $-0.33(-0.71$ to 0.04$)$ & 0.83 (0.68 to 1.02$)$ \\
\hline
\end{tabular}


Table 3| Primary and secondary outcomes for follow-up at year 1 and year 2 separately in children with recurrent upper respiratory tract infections according to treatment allocation

Adenoidectomy Watchful waiting Difference in incidence rate $(95 \% \mathrm{Cl})$ Incidence rate ratio $(95 \% \mathrm{Cl})$

Year $1^{*}$

Primary outcome:

\begin{tabular}{|c|c|c|c|c|}
\hline URTI episodes & 9.22 & 9.39 & $-0.17(-1.34$ to 1.00$)$ & $0.98(0.87$ to 1.11$)$ \\
\hline \multicolumn{5}{|l|}{ Secondary outcomes: } \\
\hline URTI days & 52.24 & 45.22 & $7.03(4.35$ to 9.71$)$ & 1.16 (1.09 to 1.22$)$ \\
\hline Severe URTI episodes & 4.47 & 4.23 & $0.25(-0.55$ to 1.05$)$ & $1.06(0.88$ to 1.27$)$ \\
\hline Severe URTI days & 39.17 & 31.39 & $7.78(5.50$ to 10.06$)$ & 1.25 (1.17 to 1.33$)$ \\
\hline Mild URTI episodes & 4.74 & 5.16 & $-0.42(-1.27$ to 0.44$)$ & $0.92(0.77$ to 1.09$)$ \\
\hline Mild URTI days & 13.07 & 13.82 & $-0.75(-2.16$ to 0.66$)$ & 0.95 (0.85 to 1.05$)$ \\
\hline Fever days & 20.78 & 16.51 & 4.27 (2.61 to 5.93 ) & 1.26 (1.15 to 1.38$)$ \\
\hline Middle ear complaints with fever episodes & 0.64 & 0.51 & $0.12(-0.17$ to 0.42$)$ & $1.24(0.75$ to 2.07$)$ \\
\hline Middle ear complaints with fever days & 1.01 & 0.88 & $0.13(-0.24$ to 0.50$)$ & $1.15(0.77$ to 1.71$)$ \\
\hline Absence from day care or school & 1.10 & 1.33 & $-0.23(-0.65$ to 0.19$)$ & $0.83(0.58$ to 1.17$)$ \\
\hline \multicolumn{5}{|l|}{ Year 2† } \\
\hline \multicolumn{5}{|l|}{ Primary outcome: } \\
\hline URTI episodes & 6.55 & 6.17 & $0.37(-0.62$ to 1.37$)$ & $1.06(0.91$ to 1.24$)$ \\
\hline \multicolumn{5}{|l|}{ Secondary outcomes: } \\
\hline URTI days & 80.60 & 91.31 & $-10.71(-14.38$ to -7.03$)$ & $0.88(0.85$ to 0.92$)$ \\
\hline Severe URTI episodes & 3.47 & 2.78 & $0.69(-0.01$ to 1.39$)$ & $1.25(1.00$ to 1.56$)$ \\
\hline Severe URTI days & 57.47 & 62.96 & $-5.50(-8.57$ to -2.42$)$ & $0.91(0.87$ to 0.96$)$ \\
\hline Mild URTI episodes & 3.08 & 3.40 & $-0.31(-1.03$ to 0.40$)$ & $0.91(0.73$ to 1.13$)$ \\
\hline Mild URTI days & 23.14 & 28.34 & $-5.21(-7.22$ to -3.20$)$ & $0.82(0.75$ to 0.88$)$ \\
\hline Fever days & 19.18 & 16.47 & $2.72(1.04$ to 4.39$)$ & $1.16(1.06$ to 1.28$)$ \\
\hline Middle ear complaints with fever episodes & 0.36 & 0.39 & $-0.03(-0.27$ to 0.22$)$ & $0.93(0.49$ to 1.78$)$ \\
\hline Middle ear complaints with fever days & 0.71 & 0.82 & $-0.11(-0.46$ to 0.23$)$ & $0.86(0.55$ to 1.36$)$ \\
\hline Absence from day care or school & 2.25 & 2.72 & $-0.47(-1.09$ to 0.16$)$ & $0.83(0.64$ to 1.07$)$ \\
\hline
\end{tabular}

URTI=upper respiratory tract infection.

*52 person years in adenoidectomy group, 53 in watchful waiting group.

†49 person years in each group. 
Table 4| Primary and secondary outcomes in children with recurrent upper respiratory tract infections according to treatment allocation adjusted for observed differences at baseline

Adenoidectomy Watchful waiting Adjusted difference in incidence rate (95\% Adjusted incidence rate ratio CI)

Total follow-up*

Primary outcome:

\begin{tabular}{|c|c|c|c|c|}
\hline URTI episodes & 7.86 & 7.89 & $-0.03(-1.72$ to 1.67$)$ & $1.00(0.80$ to 1.23$)$ \\
\hline \multicolumn{5}{|l|}{ Secondary outcomes: } \\
\hline URTI days & 66.25 & 67.20 & $-0.95(-20.50$ to 4.48$)$ & $0.99(0.73$ to 1.31$)$ \\
\hline Severe URTI episodes & 3.97 & 3.54 & $0.42(-0.63$ to 1.52$)$ & $1.12(0.84$ to 1.48$)$ \\
\hline Severe URTI days & 48.49 & 46.20 & $2.28(-15.62$ to 20.17$)$ & $1.05(0.71$ to 1.52$)$ \\
\hline Mild URTI episodes & 3.89 & 4.35 & $-0.46(-1.31$ to 0.35$)$ & $0.89(0.73$ to 1.09$)$ \\
\hline Mild URTI days & 17.80 & 21.01 & $-3.21(-7.85$ to 1.17$)$ & $0.85(0.66$ to 1.06$)$ \\
\hline Fever days & 20.16 & 16.36 & $3.80(-6.75$ to 14.20$)$ & $1.23(0.68$ to 2.12$)$ \\
\hline Middle ear complaints with fever episodes & 0.53 & 0.44 & $0.09(-0.26$ to 0.43$)$ & 1.20 (0.56 to 2.36$)$ \\
\hline Middle ear complaints with fever days & 0.91 & 0.81 & $0.10(-0.61$ to 0.77$)$ & $1.13(0.48$ to 2.60$)$ \\
\hline Absence from day care or school & 1.75 & 1.90 & $-0.15(-0.78$ to 0.48$)$ & $0.92(0.64$ to 1.30$)$ \\
\hline \multicolumn{5}{|l|}{ Year 1† } \\
\hline \multicolumn{5}{|l|}{ Primary outcome: } \\
\hline URTI episodes & 9.23 & 9.37 & $-0.14(-1.76$ to 1.68$)$ & $0.99(0.82$ to 1.20$)$ \\
\hline \multicolumn{5}{|l|}{ Secondary outcomes: } \\
\hline URTI days & 52.21 & 45.24 & $6.97(-13.24$ to 27.51$)$ & $1.15(0.74$ to 1.72$)$ \\
\hline Severe URTI episodes & 4.55 & 4.16 & $0.40(-0.68$ to 1.48$)$ & $1.10(0.85$ to 1.40$)$ \\
\hline Severe URTI days & 39.18 & 31.39 & $7.79(-11.19$ to 26.89$)$ & 1.25 (0.71 to 2.13$)$ \\
\hline Mild URTI episodes & 4.68 & 5.23 & $-0.55(-1.62$ to 0.48$)$ & $0.90(0.72$ to 1.10$)$ \\
\hline Mild URTI days & 13.03 & 13.86 & $-0.83(-5.10$ to 3.28$)$ & 0.94 (0.68 to 1.28$)$ \\
\hline Fever days & 20.70 & 16.57 & $4.13(-10.99$ to 18.38$)$ & 1.25 (0.49 to 2.66$)$ \\
\hline Middle ear complaints with fever episodes & 0.68 & 0.48 & $0.20(-0.28$ to 0.67$)$ & $1.41(0.60$ to 3.07$)$ \\
\hline Middle ear complaints with fever days & 1.09 & 0.81 & $0.28(-0.66$ to 1.18$)$ & $1.34(0.49$ to 3.61$)$ \\
\hline Absence from day care or school & 1.21 & 1.22 & $-0.01(-0.64$ to 0.61$)$ & $0.99(0.57$ to 1.63$)$ \\
\hline \multicolumn{5}{|l|}{ Year 2‡ } \\
\hline \multicolumn{5}{|l|}{ Primary outcome: } \\
\hline URTI episodes & 6.43 & 6.30 & $0.13(-2.05$ to 2.32$)$ & $1.02(0.71$ to 1.42$)$ \\
\hline \multicolumn{5}{|l|}{ Secondary outcomes: } \\
\hline URTI days & 79.60 & 89.39 & $-9.79(-35.55$ to 12.91$)$ & 0.89 (0.67 to 1.16$)$ \\
\hline Severe URTI episodes & 3.34 & 2.88 & $0.47(-0.98$ to 1.93$)$ & $1.16(0.71$ to 1.82$)$ \\
\hline Severe URTI days & 57.13 & 61.70 & $-4.57(-26.06$ to 16.37$)$ & $0.93(0.64$ to 1.32$)$ \\
\hline Mild URTI episodes & 3.08 & 3.40 & $-0.32(-1.35$ to 0.65$)$ & $0.90(0.66$ to 1.23$)$ \\
\hline Mild URTI days & 22.54 & 27.64 & $-5.10(-11.86$ to 1.39$)$ & $0.82(0.61$ to 1.05$)$ \\
\hline Fever days & 19.01 & 15.81 & $3.20(-5.09$ to 12.05$)$ & $1.20(0.73$ to 1.94$)$ \\
\hline Middle ear complaints with fever episodes & 0.35 & 0.41 & $-0.05(-0.44$ to 0.33$)$ & $0.87(0.18$ to 2.08$)$ \\
\hline Middle ear complaints with fever days & 0.71 & 0.82 & $-0.11(-0.98$ to 0.69$)$ & $0.86(0.18$ to 2.27$)$ \\
\hline Absence from day care or school & 2.31 & 2.57 & $-0.26(-1.24$ to 0.73$)$ & $0.90(0.59$ to 1.35$)$ \\
\hline
\end{tabular}

URTI=upper respiratory tract infection.

*101 person years in each group.

†52 person years in adenoidectomy group, 53 in watchful waiting group.

$\ddagger 49$ person years in each group. 
Table 5| Results for per protocol and as treated analyses for full follow-up period (maximum 24 months) in children with recurrent upper respiratory tract infections

Adenoidectomy Watchful waiting Difference in incidence rate $(95 \% \mathrm{Cl})$ Incidence rate ratio $(95 \% \mathrm{Cl})$

\section{Per protocol ${ }^{*}$}

\begin{tabular}{|c|c|c|c|c|}
\hline \multicolumn{5}{|l|}{ Primary outcome: } \\
\hline URTI episodes & 7.91 & 8.04 & $-0.13(-1.02$ to 0.77$)$ & $0.98(0.88$ to 1.10$)$ \\
\hline \multicolumn{5}{|l|}{ Secondary outcomes: } \\
\hline URTI days & 66.10 & 57.63 & 8.47 (5.99 to 10.95$)$ & $1.15(1.10$ to 1.19$)$ \\
\hline Severe URTI episodes & 3.98 & 3.50 & $0.48(-0.13$ to 1.09$)$ & $1.14(0.96$ to 1.34$)$ \\
\hline Severe URTI days & 48.11 & 35.42 & $12.69(10.67$ to 14.70$)$ & $1.36(1.29$ to 1.43$)$ \\
\hline Mild URTI episodes & 3.93 & 4.54 & $-0.60(-1.26$ to 0.06$)$ & $0.87(0.74$ to 1.01$)$ \\
\hline Mild URTI days & 17.99 & 22.21 & $-4.22(-5.66$ to -2.77$)$ & $0.81(0.76$ to 0.87$)$ \\
\hline Fever days & 20.00 & 15.11 & 4.88 (3.58 to 6.19$)$ & $1.32(1.22$ to 1.43$)$ \\
\hline Middle ear complaints with fever episodes & 0.51 & 0.46 & $0.05(-0.17$ to 0.27$)$ & $1.10(0.69$ to 1.75$)$ \\
\hline Middle ear complaints with fever days & 0.86 & 0.87 & $-0.10(-0.39$ to 0.19$)$ & $0.89(0.63$ to 1.26$)$ \\
\hline Absence from day care or school & 1.66 & 1.83 & $-0.17(-0.59$ to 0.25$)$ & $0.91(0.71$ to 1.15$)$ \\
\hline \multicolumn{5}{|l|}{ As treated $\dagger$} \\
\hline \multicolumn{5}{|l|}{ Primary outcome: } \\
\hline URTI episodes & 7.81 & 8.04 & $-0.23(-1.08$ to 0.62$)$ & $0.97(0.87$ to 1.08$)$ \\
\hline \multicolumn{5}{|l|}{ Secondary outcomes: } \\
\hline URTI days & 70.67 & 57.63 & 13.04 (10.69 to 15.40$)$ & 1.23 (1.18 to 1.27$)$ \\
\hline Severe URTI episodes & 3.93 & 3.50 & $0.42(-0.15$ to 1.00$)$ & $1.12(0.96$ to 1.31$)$ \\
\hline Severe URTI days & 52.49 & 35.42 & 17.07 (15.16 to 18.99$)$ & 1.48 (1.41 to 1.55$)$ \\
\hline Mild URTI episodes & 3.94 & 4.54 & $-0.59(-1.22$ to 0.03$)$ & $0.87(0.75$ to 1.00$)$ \\
\hline Mild URTI days & 18.18 & 22.21 & $-4.03(-5.40$ to -2.65$)$ & $0.82(0.77$ to 0.87$)$ \\
\hline Fever days & 19.59 & 15.11 & 4.48 (3.26 to 5.70$)$ & $1.30(1.20$ to 1.40$)$ \\
\hline Middle ear complaints with fever episodes & 0.49 & 0.46 & $0.03(-0.17$ to 0.24$)$ & 1.07 (0.69 to 1.66$)$ \\
\hline Middle ear complaints with fever days & 0.85 & 0.87 & $-0.02(-0.30$ to 0.26$)$ & $0.98(0.71$ to 1.35$)$ \\
\hline Absence from day care or school & 1.83 & 1.83 & $0.00(-0.41$ to 0.40$)$ & $1.00(0.80$ to 1.25$)$ \\
\hline
\end{tabular}

URTI=upper respiratory tract infection.

*101 person years in adenoidectomy group, 61 in watchful waiting group.

†141 person years in adenoidectomy group, 61 in watchful waiting group. 
Table $6 \mid$ Results for per protocol and as treated analyses adjusted for observed baseline differences for full follow-up period (maximum 24 months) in children with recurrent upper respiratory tract infections

Adenoidectomy Watchful waiting Difference in incidence rate $(95 \% \mathrm{Cl})$ Incidence rate ratio $(95 \% \mathrm{Cl})$

\section{Per protocol ${ }^{*}$}

\begin{tabular}{|c|c|c|c|c|}
\hline \multicolumn{5}{|l|}{ Primary outcome: } \\
\hline URTI episodes & 7.89 & 8.01 & $-0.19(-2.27$ to 1.83$)$ & $0.97(0.75$ to 1.26$)$ \\
\hline \multicolumn{5}{|l|}{ Secondary outcomes: } \\
\hline URTI days & 66.23 & 57.44 & $8.78(-12.98$ to 30.34$)$ & $1.15(0.81$ to 1.63$)$ \\
\hline Severe URTI episodes & 3.97 & 3.53 & $0.44(-0.82$ to 1.72$)$ & $1.12(0.81$ to 1.60$)$ \\
\hline Severe URTI days & 48.34 & 35.15 & $13.19(-6.31$ to 32.62$)$ & $1.38(0.86$ to 2.23$)$ \\
\hline Mild URTI episodes & 3.92 & 4.56 & $-0.63(-1.68$ to 0.33$)$ & $0.86(0.67$ to 1.08$)$ \\
\hline Mild URTI days & 17.89 & 22.40 & $-4.58(-10.31$ to 0.72$)$ & $0.80(0.60$ to 1.04$)$ \\
\hline Fever days & 20.36 & 14.69 & $5.67(-4.76$ to 15.43$)$ & $1.39(0.77$ to 2.45$)$ \\
\hline Middle ear complaints with fever episodes & 0.52 & 0.44 & $0.08(-0.36$ to 0.47$)$ & $1.18(0.50$ to 3.08$)$ \\
\hline Middle ear complaints with fever days & 0.90 & 0.81 & $0.09(-0.91$ to 0.87$)$ & $1.11(0.41$ to 3.84$)$ \\
\hline Absence from day care or school & 1.73 & 1.72 & $0.01(-0.71$ to 0.74$)$ & $1.01(0.67$ to 1.56$)$ \\
\hline \multicolumn{5}{|l|}{ As treated $\dagger$} \\
\hline \multicolumn{5}{|l|}{ Primary outcome: } \\
\hline URTI episodes & 7.78 & 8.10 & $-0.31(-2.16$ to 1.47$)$ & $0.96(0.77$ to 1.21$)$ \\
\hline \multicolumn{5}{|l|}{ Secondary outcomes: } \\
\hline URTI days & 71.02 & 56.98 & $14.04(-4.35$ to 31.87$)$ & 1.25 (0.94 to 1.68$)$ \\
\hline Severe URTI episodes & 3.84 & 3.55 & $0.29(-0.82$ to 1.39$)$ & $1.08(0.81$ to 1.49$)$ \\
\hline Severe URTI days & 52.84 & 34.90 & $17.93(0.34$ to 34.44$)$ & 1.51 (1.02 to 2.33$)$ \\
\hline Mild URTI episodes & 3.94 & 4.54 & $-0.60(-1.56$ to 0.32$)$ & $0.87(0.70$ to 1.08$)$ \\
\hline Mild URTI days & 18.19 & 22.17 & $-3.99(-9.48$ to 1.13$)$ & $0.82(0.63$ to 1.06$)$ \\
\hline Fever days & 19.71 & 14.90 & $4.81(-4.42$ to 13.28$)$ & $1.32(0.78$ to 2.23$)$ \\
\hline Middle ear complaints with fever episodes & 0.49 & 0.45 & $0.04(-0.34$ to 0.37$)$ & $1.10(0.53$ to 2.63$)$ \\
\hline Middle ear complaints with fever days & 0.86 & 0.84 & $0.02(-0.87$ to 0.72$)$ & $1.03(0.44$ to 3.30$)$ \\
\hline Absence from day care or school & 1.87 & 1.75 & $0.12(-0.54$ to 0.75$)$ & $1.07(0.75$ to 1.56$)$ \\
\hline
\end{tabular}

URTI=upper respiratory tract infection.

*101 person years in adenoidectomy group, 61 in watchful waiting group.

†141 person years in adenoidectomy group, 61 in watchful waiting group. 


\section{Figures}

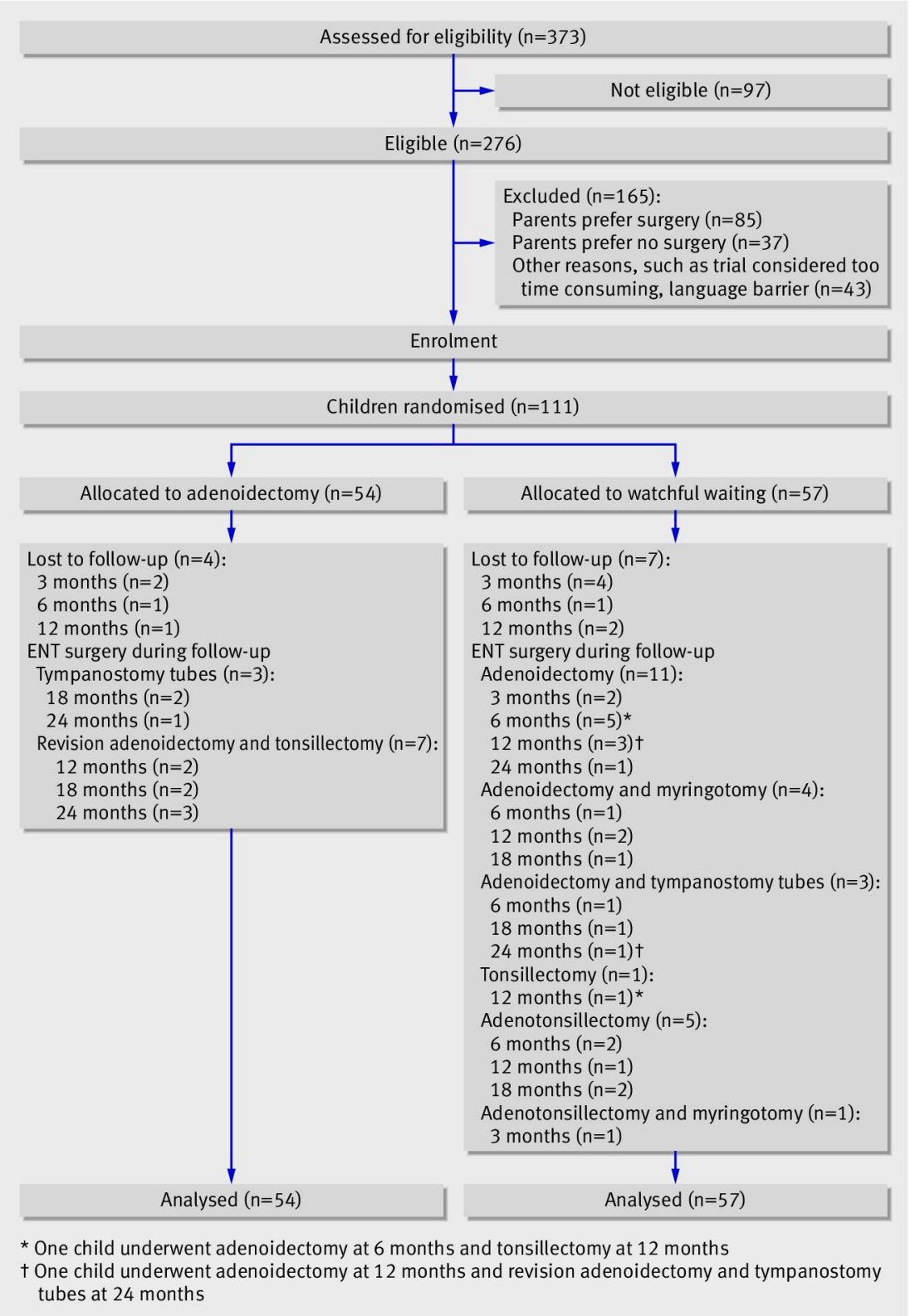

Fig 1 Flow of participants through trial of adenoidectomy in children with recurrent upper respiratory tract infections

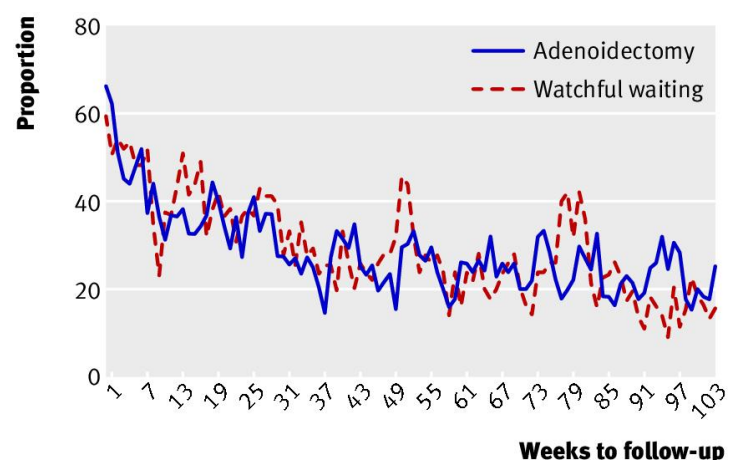

Fig 2 Proportion of children with upper respiratory tract infection (prevalence/week) in adenoidectomy and watchful waiting group 